\section{Enseñar y aprender a leer en inglés: creencias de futuros docentes de lengua extranjera chilenos*}

Tania Tagle Ochoa** https://orcid.org/0000-0002-0299-1827

Cecilia Schuster Muñoz https://orcid.org/0000-0002-5689-2592

Viviana Rojas Caro ${ }^{* * * *}$ https://orcid.org/0000-0001-6297-1150

Mónica Campos Espinoza ${ }^{* * * * * *}$ https://orcid.org/0000-0002-1681-8910

Lucía Ubilla Rosales ${ }^{* * * * *}$ https://orcid.org/0000-0001-9933-7997

Luzmila Flores Gajardo ${ }^{* * * * * *}$ https://orcid.org/0000-0003-1296-4635

Mark Briesmaster ${ }^{* * * * * * *}$ https://orcid.org/0000-0001-5078-3718

* Este artículo forma parte del Proyecto de Innovación en Docencia 411-3462.

** Académica de la carrera de Pedagogía en inglés. Universidad Católica de Temuco, Temuco, Chile. Correo electrónico: ttagle@uct.cl.

*** Académica de la carrera de Pedagogía en inglés. Universidad Católica de Temuco, Temuco, Chile. Correo electrónico: cschuster@uct.cl.

**** Académica de la carrera de Pedagogía en inglés. Universidad Católica de Temuco, Temuco, Chile. Correo electrónico: vrojas@uct.cl.

**** Académica de la carrera de Pedagogía en inglés. Universidad Católica de Temuco, Temuco, Chile. Correo electrónico: mcampos@uct.cl.

****** Académica de la carrera de Pedagogía en inglés. Universidad Católica de Temuco, Temuco, Chile. Correo electrónico: lubilla@uct.cl.

******* Académica de la carrera de Pedagogía en inglés. Universidad Católica de Temuco, Temuco, Chile. Correo electrónico: Iflores@uct.cl.

******* Académico de la carrera de Pedagogía en inglés. Universidad Católica de Temuco, Temuco, Chile. Correo electrónico: briesmaster@uct.cl.

\author{
Ensinar e aprender a \\ ler em inglês: Crenças \\ chilenos de língua \\ estrangeira
}
de futuros professores
Teaching and
Service
Beliefs

FOLIOS

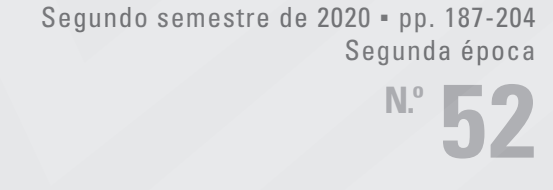




\section{Resumen}

El objetivo del presente artículo derivado de investigación es develar las creencias sobre el proceso de enseñanza y aprendizaje de la habilidad de comprensión lectora que poseen estudiantes de Pedagogía en inglés de una universidad chilena. Se siguió una metodología cualitativa de investigación, empleando un estudio de caso. Los participantes fueron 20 futuros docentes, que estaban cursando su segundo año de formación. Se recogió información a través de una entrevista semiestructurada. Los datos se analizaron utilizando el software Atlas. Ti. Los resultados plantean que los participantes poseen creencias sobre esta habilidad basadas en las formas de procesamiento ascendente y descendente de la información. Al respecto, se señala que los futuros maestros requieren configurar una visión sobre la comprensión lectora desde una perspectiva estratégica. En este contexto, se sugiere que las instituciones formadoras de profesores de inglés los incentiven a realizar prácticas metacognitivas para transformar sus creencias tradicionales.

\section{Palabras clave}

Formación de docentes; creencias; comprensión lectora; enseñanza de idiomas; aprendizaje de idiomas

Abstract

The objective of the present article derived from research is to unveil the beliefs about the teaching and learning process of the reading comprehension skill that students of Pedagogy in English possess at a Chilean university. The authors followed a qualitative research methodology through a case study. The participants were 20 future teachers, who were in their second year of education. The authors conducted a semi-structured interview and analyzed the participants' answers using the Atlas. Ti software. The findings suggest that the participants hold beliefs concerning reading skills in English which are based on both bottom-up and top-down ways of processing information. In this respect, the authors state that future teachers need to build up a new look at reading comprehension from a strategic approach. In this context, it is suggested that English

teacher training institutions encourage them to carry out metacognitive practices to transform their traditional beliefs.

\section{Keywords}

teacher education; beliefs; reading comprehension; language teaching; language learning

\section{Resumo}

0 objetivo do presente estudo é revelar as crenças sobre o processo de ensino e aprendizagem das habilidades de compreensão de leitura que têm alunos de Pedagogia em inglês de uma universidade chilena. A metodologia de pesquisa qualitativa foi considerada, utilizando um estudo de caso. Os participantes foram 20 futuros professores, estando em seu segundo ano de formação. As informações foram coletadas por meio de uma entrevista semiestruturada. Os dados foram analisados utilizando o software Atlas. Ti. Os resultados sugerem que os participantes têm crenças sobre essa habilidade com base nas formas de processamento de informações ascendente e descendente. Sugere-se que as instituições que treinam professores de inglês os incentivem a realizar práticas metacognitivas para transformar suas crenças tradicionais.

\section{Palavras-chave}

formação de professores; crenças; compreensão de leitura; ensino de línguas; aprendizagem de línguas 


\section{Introducción}

El sistema educacional chileno considera como asignatura obligatoria la enseñanza del inglés como lengua extranjera desde el quinto año de educación primaria hasta el cuarto año de educación secundaria (Ministerio de Educación de Chile, 2012). Al respecto, las metas que los estudiantes deberían alcanzar durante su proceso formativo se basan en niveles de inglés asociados a estándares internacionales, los cuales, en este caso, son los planteados por el Marco Común Europeo de Referencia para las Lenguas. Estos niveles son "A1 (Acceso), A2 (Plataforma), B1 (Umbral), B2 (Avanzado), C1 (Dominio operativo eficaz) y C2 (Maestría)" (Consejo de Europa, 2001, p. 28). En este marco, se espera que los estudiantes chilenos, al finalizar su educación primaria, hayan alcanzado un nivel de inglés A2. Asimismo, se contempla que ellos deberían contar con un nivel B1 al egresar de educación secundaria (Ministerio de Educación de Chile, 2014).

En el contexto nacional, el Sistema de Medición para la Calidad de la Educación (Simce), por medio de un examen de inglés focalizado en las habilidades de comprensión lectora y comprensión auditiva busca recoger información sobre el nivel que poseen los alumnos de tercer año de enseñanza secundaria en función de estas competencias receptivas (Agencia de Calidad de la Educación, 2015). Los resultados de la última evaluación aplicada el año 2014 indicaron que solo un 12,6\% de los aprendices logra demostrar un B1 de acuerdo con el Marco Común Europeo de Referencia para las Lenguas al comprender de manera escrita y oral en inglés. Estos hallazgos parecen evidenciar que los estudiantes chilenos finalizan su formación escolar experimentando dificultades para comprender textos escritos en la lengua inglesa, lo cual resulta preocupante en el marco de las metas establecidas por el Ministerio de Educación.

Uno de los factores que podría estar limitando el aprendizaje de la comprensión lectora en inglés de los estudiantes chilenos correspondería a las ideas de los docentes sobre el aprendizaje y la enseñanza de esta lengua. En esta línea, algunos estudios llevados a cabo en el país han encontrado que profesores de inglés, en formación y en ejercicio, sostienen que el aprendizaje de este idioma se construye a través de la formación de hábitos o memorización considerando contenidos lingüísticos, tales como gramática y vocabulario, por sobre el desarrollo de las habilidades comunicativas (Blázquez y Tagle, 2010; Tagle, Díaz, Alarcón, Quintana y Ramos, 2014a).

Por otro lado, estudios similares llevados a cabo en Chile establecen que los docentes de inglés, en formación y en ejercicio, vinculan la enseñanza de la lengua extranjera con procesos de transmisión de información más que con proveer las condiciones necesarias para generar el desarrollo de habilidades receptivas y productivas por parte de los estudiantes (Díaz, Alarcón y Ortiz, 2012, 2015; Tagle, Díaz, Alarcón, Quintana y Ramos, 2014b). La visión de los profesionales de la educación sobre cómo ayudar a otros a construir conocimientos respecto a la lengua inglesa se asociaría a sus creencias sobre el proceso de enseñanza y aprendizaje en el área de especialidad. Dicho proceso se asociaría, a su vez, con algunas dimensiones didácticas, entre ellas: objetivos, contenidos, actividades, recursos y formas de evaluación (Zabala, 2007). De acuerdo con Borg (2006), las creencias pedagógicas corresponden a la forma como los docentes perciben e interpretan los procesos de aprendizaje y enseñanza. Por lo tanto, las mismas tenderán a conducir, de manera directa, el desempeño de los profesionales de la educación.

En este contexto, considerando los bajos resultados alcanzados por los alumnos chilenos en el marco de la habilidad de comprensión lectora en inglés, parece importante develar las creencias sobre el proceso de enseñanza y aprendizaje de esta habilidad que poseen futuros profesores de lengua inglesa pertenecientes a una universidad nacional. Específicamente, esta investigación busca responder las siguientes preguntas de investigación:

1. ¿Cuáles son las creencias sobre objetivos y contenidos asociados a la habilidad de comprensión lectora que poseen futuros profesores de inglés que cursan su segundo año de formación en una universidad chilena? 
2. ¿Cuáles son las creencias sobre actividades y recursos relacionados con la habilidad de comprensión lectora que poseen futuros profesores de inglés que cursan su segundo año de formación en una universidad chilena?

3. ¿Cuáles son las creencias sobre evaluación respecto a la habilidad de comprensión lectora que poseen futuros profesores de inglés que cursan su segundo año de formación en una universidad chilena?

\section{Marco teórico}

\section{Creencias pedagógicas}

En términos generales, es posible definir las creencias como constructos mentales, los cuales funcionan como un filtro de información (Díaz y Solar, 2008; Pajares, 1992). En el contexto pedagógico, las creencias pueden definirse como teorías personales y representaciones que cada docente posee con respecto al proceso de enseñanza y aprendizaje (Levin y He, 2008). Al respecto, se ha indicado que las creencias pedagógicas influencian el actuar del docente en el aula (Borg, 2011); por consiguiente, son las que determinan cómo enseñar a los estudiantes.

Considerando el proceso de construcción de las creencias, los individuos las desarrollan de manera inconsciente e involuntaria (Cross, 2009; Inceday y Kesli, 2011; Richardson, 2003). De acuerdo con estos autores, las creencias suelen construirse durante la formación de los sujetos a partir de la observación de las prácticas pedagógicas de los docentes en el contexto escolar. Relativo a este punto, Mak (2011) sostiene que las creencias construidas en este tipo de contextos son muy difíciles de modificar.

Ertmer (2005) expresa que las creencias se encuentran arraigadas o centralizadas en la cognición de los profesionales de la educación. Por esta razón, el hecho de querer modificarlas resulta un proceso muy complejo. En la misma línea, Pajares (1992) sostiene que las creencias internalizadas a más temprana edad resultan ser más difíciles de modificar que las adquiridas recientemente. Esto se debería a que las creencias más antiguas están mayormente enraizadas en lo profundo de la cognición del individuo. Otro factor que afecta la modificación de las creencias se asocia a su naturaleza tácita (Erkmen, 2014), es decir, tienen un carácter implícito, por lo que es muy complejo articularlas.

Se ha indicado que las creencias pueden modificarse, de manera progresiva. Al respecto, Richards y Farrell (2005) plantean que los procesos de reflexión sobre la práctica pueden contribuir a este cambio. En esta línea, cuestionar las propias acciones docentes, articulando la teoría y la práctica, puede promover la transformación de creencias que no favorecen la implementación de procesos de enseñanza y aprendizaje innovadores. Lo anterior, porque las creencias filtran la información que proviene del entorno y/o la adecúan considerando la tradición.

\section{Habilidad de comprensión lectora en inglés}

La comprensión lectora en inglés es una habilidad receptiva, a través de la cual los individuos procesan e identifican información de textos escritos (Grabe, 2009). Este proceso comprende la construcción activa de significados respecto a elementos textuales y contextuales. En este marco, la puesta en práctica de esta habilidad puede asociarse a diferentes propósitos, tales como localizar, integrar, criticar y usar información, comprender de manera rápida y general, además de aprender y evaluar.

Considerando la habilidad de comprensión lectora en inglés, se plantean dos formas de procesamiento de la información. La primera es la de tipo ascendente o bottom-up (Carrell, 1987; Kusiak, 2013). Desde este punto de vista, la comprensión lectora se percibe como un proceso de decodificación de información. Al respecto, se comienza decodificando unidades lingüísticas individuales del texto, desde las que son mínimas hasta las de mayor extensión. En otras palabras, un lector comprende un texto decodificando, primero, letras, luego el significado de palabras y, posteriormente, el de un párrafo completo, repitiendo el mismo proceso para los componentes restantes del texto. 
La segunda forma de procesamiento de la información usada al comprender un texto escrito es la de tipo descendente o top-down (Dambacher, 2010; Smith, 1978), que se basa en un modelo psicolingüístico que estipula Goodman (1988). En esta forma de procesamiento, la comprensión lectora corresponde a un proceso activo, donde un lector, para entender un texto, realiza predicciones y activa sus conocimientos previos. En esta línea, la comprensión desde esta lógica integra factores asociados al lector, tales como sus experiencias, sus perspectivas y conocimiento cultural (Nunan, 2015). Además, esta forma de procesamiento se centra en componentes que tienen relación con el texto, tales como temáticas, estructuras textuales y secuencias episódicas.

En este contexto, existe consenso en que la habilidad de comprensión lectora se produce a través de la confluencia de las formas de procesamiento ascendente y descendente, es decir, a través de un proceso interactivo relacionado con las formas de procesamiento previamente identificadas (Hedgcock y Ferris, 2009). Desde esta perspectiva, se sugiere no basarse en una lógica exclusiva, sino considerar las dos formas de procesamiento como complementarias más que opuestas.

\section{Dimensiones didácticas involucradas en la enseñanza y el aprendizaje del inglés}

El diseño y la implementación del proceso de enseñanza y aprendizaje de la lengua inglesa se basan, esencialmente, en tomar decisiones teniendo en cuenta determinadas dimensiones didácticas (Ministerio de Educación de Chile, 2012) que se asocian a objetivos, contenidos, actividades, recursos y evaluación.

Respecto a los objetivos de aprendizaje, estos corresponden a metas que estipulan los resultados que los aprendices deben lograr al finalizar una actividad académica (Phillips y Pulliam Phillips, 2008). En el contexto de la enseñanza del inglés, los objetivos de aprendizaje pueden relacionarse con el desarrollo de la competencia comunicativa de esta lengua extranjera (Canale, 1983; Canale y Swain, 1980; Celce-Murcia, Dörnyei y Thurrell, 1995;
Celce-Murcia y Olshtain, 2000; Savignon, 2001). Al respecto, Usó-Juan y Martínez-Flor (2006), basándose en el trabajo de los autores citados, proponen un modelo de competencia comunicativa compuesto por las siguientes cinco áreas de competencia: competencia discursiva, lingüística, pragmática, intercultural y estratégica. Al respecto, la competencia discursiva se relaciona con la comprensión y producción de textos en función de las habilidades de comprensión lectora y auditiva, expresión escrita y oral. Lo anterior implica la coherencia y cohesión en diversos tipos de textos. La competencia lingüística se asocia a todos los elementos de un sistema lingüístico: fonológicos, gramaticales y léxicos, que son necesarios para comprender y producir un texto oral o escrito. Asimismo, la competencia pragmática se enmarca en conocer la función de un texto escrito u oral, además de los factores contextuales implicados. Por su parte, la competencia intercultural se relaciona con el conocimiento de reglas socioculturales sobre cómo comprender o producir un texto oral o escrito coherente con determinados contextos sociolingüísticos. De acuerdo con estos autores, el quinto componente de la competencia comunicativa es la competencia estratégica, que se asocia al conocimiento de estrategias compensatorias en caso de que el usuario del idioma experimente dificultades respecto a las competencias previamente descritas.

Los objetivos se asocian a los contenidos o saberes que los estudiantes han de construir los cuales pueden ser de cuatro tipos: contenidos factuales, conceptuales, procedimentales y actitudinales (López, 2005; Zabala, 2007; Zabala y Arnau, 2014). Los primeros se refieren a información literal relacionada con datos, hechos o términos, y tienden a ser memorizados. Por su parte, los contenidos conceptuales también se refieren a los aspectos mencionados, pero buscando comprenderlos. En tercer lugar, los contenidos procedimentales corresponden al saber hacer, lo cual se vincula con habilidades, técnicas y estrategias que los estudiantes adquirirán. Finalmente, los contenidos de tipo actitudinal apuntan a un saber ser, centrándose especialmente en normas, valores y actitudes. 
Asociadas a la enseñanza de la habilidad de comprensión lectora se sugieren tres tipos de tareas: las actividades previas a la lectura, las actividades durante la lectura y las actividades posteriores a la lectura. En las primeras, se prepara al estudiante para comprender un texto, activando sus conocimientos, presentando vocabulario clave y ayudándolo a hacer predicciones sobre la temática del texto (Makalela, 2015). En las actividades realizadas durante la lectura, el docente acompaña a los estudiantes, incentivándolos a comprender los textos con el fin de identificar información general para confirmar sus predicciones y, simultáneamente, identificar información específica para reconocer detalles en el texto (Grabe y Stoller, 2014). Respecto a las actividades posteriores a la lectura, estas permiten profundizar la comprensión de los textos y/o llevar a cabo la producción del idioma (Xiao, 2015).

En cuanto a los recursos, Tomlinson (2011) señala que estos pueden tratarse de cualquier material usado para ayudar a otros a adquirir la lengua inglesa. En el marco de la enseñanza de la habilidad de comprensión lectora en inglés, Maley (2011) indica que se usan textos no literarios y literarios para exponer a los estudiantes al idioma extranjero. Por un lado, es posible hacer alusión a los textos no literarios, que buscan informar o persuadir respecto a acontecimientos de la vida cotidiana (Ramírez, Rossel y Nazar, 2015). Ejemplos de estos pueden ser noticias, afiches, cartas, entrevistas y textos normativos. Por otro lado, los textos literarios presentan hechos relacionados con la ficción (Mullis, Kennedy, Martin y Sainsbury, 2006). Se ha señalado que los mismos son recursos auténticos que exponen el idioma de manera no adaptada a los aprendices (Alonso y Fernández, 2003). Ejemplos de este tipo de textos son poemas, historias breves o novelas (Richards, 2015).

Respecto a los tipos de textos, Rodríguez (2012) señala que estos pueden tener distintas estructuras; se clasifican en narrativos, descriptivos, expositivos, argumentativos, dialogados e instructivos. De acuerdo con esta autora, los textos narrativos presentan eventos, ficticios o reales, llevados a cabo por personajes. Los textos descriptivos buscan representar con detalle cosas, animales, paisajes, épocas o personas. Los textos expositivos exponen conceptos o ideas, considerando un orden lógico que puede ser desde lo más general a lo más particular (orden deductivo), desde lo más particular a lo más general (inductivo) o por medio de ideas sucesivas (orden paralelístico). Los textos argumentativos incluyen la formulación de hipótesis o tesis que permiten la demostración de un juicio. Los textos dialogados involucran las interacciones de dos o más personajes. Por último, de acuerdo con esta autora, los textos instructivos buscan aconsejar, dirigir o enseñar algo al receptor, combinando la descripción y la exposición.

En el contexto de la evaluación en la enseñanza del inglés, esta se asocia a verificar el logro de los objetivos de aprendizaje por parte de los aprendices (Brown y Abeywickrama, 2010). Al respecto, se destacan tres tipos de evaluación: diagnóstica, formativa y sumativa (Bachman y Palmer, 2010; Katz, 2014). La primera se lleva a cabo para identificar los conocimientos previos de los aprendices al inicio de un determinado periodo académico previo a cada diseño áulico. La formativa se realiza durante el proceso de aprendizaje a través de las actividades de clase y retroalimentación. La evaluación sumativa se implementa al final de una unidad o curso para calificar el nivel de logro de los objetivos de aprendizaje por parte de los estudiantes.

En función de la evaluación asociada a la habilidad de comprensión lectora, pueden considerarse actividades abiertas, que buscan que el estudiante produzca una respuesta en función de lo comprendido de un texto escrito (Oakhill, Cain y Elbro, 2015). De la misma forma, se puede evaluar la comprensión lectora por medio de actividades cerradas, en las cuales el estudiante debe demostrar lo que ha entendido sobre un texto, seleccionando una respuesta previamente definida, como en el caso de tareas de completar, selección múltiple o verdadero-falso (Alderson, 2000).

\section{Metodología}

En el presente estudio se siguió una metodología cualitativa de investigación, debido a que es coherente con el reconocimiento de dimensiones subjetivas y conocimiento personal de los participantes. En este sentido, es importante señalar que el estudio 
de las creencias se reconoce como relacionado, esencialmente, con la metodología cualitativa, por la naturaleza de estas y por estar estrechamente conectadas con el pensamiento (Sandín, 2003). En este marco, se empleó un análisis de caso centrado en las creencias que poseían futuros profesores de una universidad chilena. El estudio de casos se entiende como aquel que se centra en la particularidad y en la complejidad de un caso singular, para comprenderlo en las circunstancias en que se desarrolla (Stake, 1998). El estudio de casos se caracteriza por ser particularista, descriptivo y heurístico porque se centra en una situación, acontecimiento o fenómeno en particular; se describe de manera precisa y completa el fenómeno estudiado; y permite darle nuevos significados.

\section{Participantes}

Los sujetos de estudio fueron 20 estudiantes del segundo año de la carrera Pedagogía en inglés de una universidad chilena ubicada en el sur del país. Del total de participantes, 10 eran mujeres y 10 hombres, y sus edades fluctuaban entre los 20 y 25 años.

Como criterios de inclusión, se consideró que los participantes estuviesen cursando su cuarto semestre de formación universitaria (la duración total del programa es de 10 semestres académicos). En este semestre a ellos aún no les corresponde asistir a cursos asociados a didáctica de la especialidad o asignaturas en donde reflexionen sobre la enseñanza de la habilidad de comprensión lectora en inglés. Se realizó una invitación vía directa a los estudiantes en formación para colaborar en el estudio; la participación de los sujetos fue voluntaria.

\section{Instrumento de recolección de datos}

El instrumento utilizado para recolectar información fue una entrevista semiestructurada (véase el anexo). Esta técnica fue seleccionada para que los participantes pudiesen explicitar, de manera oral, y dialogar con el entrevistador en el marco de sus creencias respecto a objetivos, contenidos, actividades, recursos y evaluación centrados en la habilidad de comprensión lectora en inglés. Para facilitar el acceso a la información, se decidió realizar la entrevista en español (idioma materno de los participantes). El guion de la entrevista fue construido por los investigadores y validado a través de juicio de expertos.

\section{Procedimiento}

En primer lugar, se invitó como participantes a futuros profesores de inglés que estuvieran cursando el segundo año de formación en una universidad chilena. Las personas que accedieron firmaron una carta de consentimiento, la cual explicitaba que los datos recogidos solo serían útiles para fines investigativos y que no se revelarían sus identidades.

Como paso siguiente, uno de los investigadores entrevistó a los participantes. Cada entrevista duró alrededor de 30 minutos y se registró por medio de una grabadora de audio. Posteriormente, las entrevistas se transcribieron usando el programa procesador de texto Microsoft Word. Estas transcripciones fueron analizadas por medio del software de análisis cualitativo Atlas. Ti. Las respuestas de los sujetos fueron codificadas y categorizadas, con el apoyo del software, considerando las dimensiones didácticas de objetivos, contenidos, actividades, recursos y evaluación asociadas a la habilidad de comprensión lectora en inglés. Por último, se procedió a crear redes conceptuales, incluyendo las categorías previamente establecidas, para ilustrar los hallazgos del estudio.

\section{Resultados}

\section{Creencias de futuros profesores de inglés sobre objetivos y contenidos asociados a la habilidad de comprensión lectora}

La categoría Creencias de futuros profesores de inglés sobre objetivos asociados a la habilidad de comprensión lectora, que se ilustra en la figura 1, permite identificar, por un lado, las subcategorías identificar información y aprender contenidos lingüísticos. Asimismo, la categoría Creencias de futuros profesores de inglés sobre contenidos asociados a la habilidad de comprensión lectora, también expuesta en la figura 1, permite identificar las subcategorías contenidos conceptuales y contenidos procedimentales. 


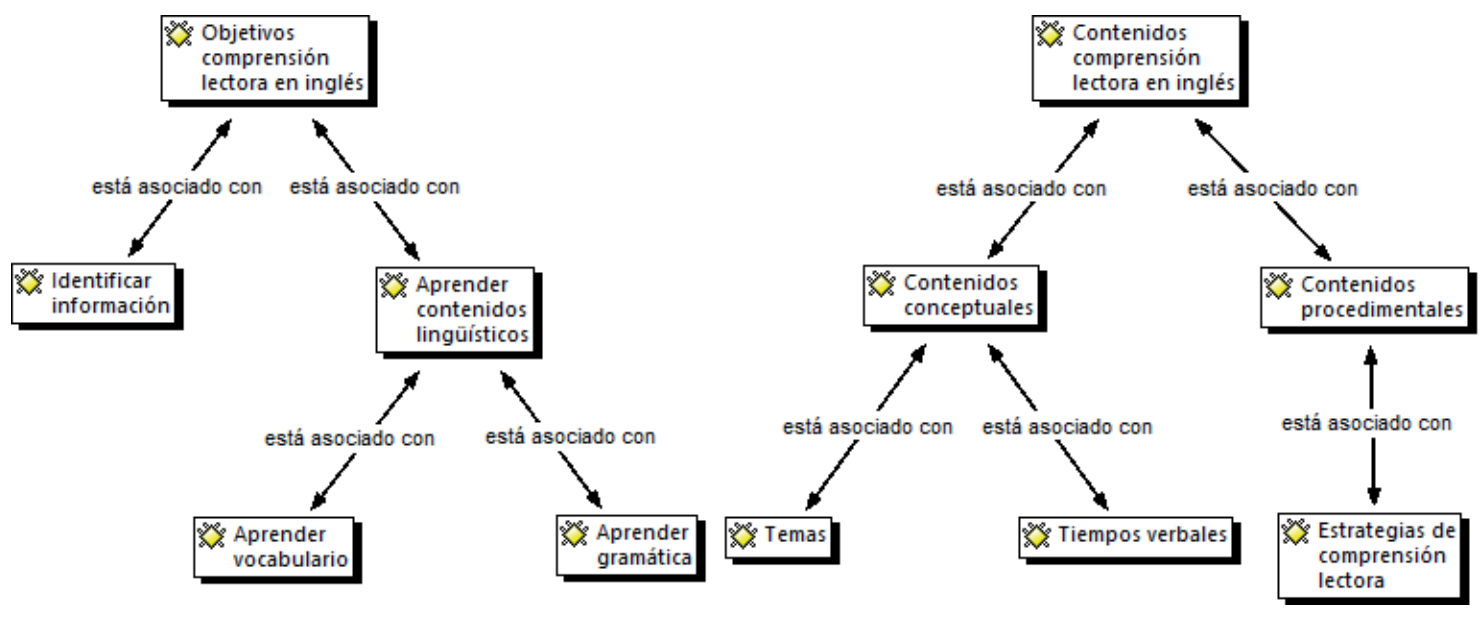

Figura 1. Red conceptual asociada a Creencias de futuros profesores de inglés sobre objetivos y contenidos asociados a la habilidad de comprensión lectora.

Fuente: elaboración propia.

En el marco de las creencias focalizadas en objetivos de la habilidad de comprensión lectora en inglés que poseen los estudiantes de pedagogía, uno de los propósitos mencionados por los participantes en la entrevista fue identificar información en textos. Lo anterior se explicita en el siguiente segmento de entrevista: "Los alumnos deben extraer información global, información específica, detalles, palabras específicas, vocabulario del texto" (E07 [13:13]). En la misma línea, otro de los informantes manifiesta una perspectiva similar: "Los alumnos deberían lograr una comprensión completa de lo que se lee identificando información general, información específica y analizando diferentes partes del texto que son inicio, desarrollo y final" (E08 [11:11]).

Además, algunos estudiantes de pedagogía expresan que uno de los objetivos relacionados con la habilidad de comprensión lectora en inglés debería ser aprender contenidos lingüísticos. En este contexto, los entrevistados manifiestan que los estudiantes de la lengua inglesa deberían aprender vocabulario para poder reconocer lo señalado en los textos. Al respecto, uno de los informantes establece lo siguiente: "Primero se aprende lo más básico que es el vocabulario. Esto se hace traduciendo las palabras. Se deben conocer de forma aislada para comprender cada oración del texto" (E06 [05:05]). Esta visión también se evidencia en: "Una clase de lectura pudiese ser útil para que los alumnos adquieran vocabulario o palabras específicas incluidas en los textos" (E09 [15:15]).

Por otro lado, considerando el objetivo aprender contenidos lingüísticos, los futuros docentes manifiestan que los alumnos, al desarrollar la habilidad de comprensión lectora en inglés, deberían aprender gramática, lo cual involucra adquirir saberes estructurales de la lengua inglesa. En este contexto, uno de los participantes plantea que: "Al leer en inglés, el estudiante pudiese reconocer cómo se estructura el idioma. Uno no siempre piensa en eso" (E10 [17:17]). Este punto de vista es también explicitado por otro de los sujetos de estudio: "Uno de los objetivos que los alumnos deberían alcanzar, cuando leen textos, es aprender estructuras gramaticales para producirlas más adelante" (E07 [13:13]).

Las creencias de los futuros docentes de inglés se focalizan, además, en ciertos contenidos respecto a la habilidad de comprensión lectora. Desde esta perspectiva, al hacer alusión a los saberes que los aprendices deberían construir al desarrollar esta habilidad, los informantes se refirieron a contenidos conceptuales. Los mismos se asocian a principios, hechos o datos que deben comprenderse. Uno de estos corresponde a distintos temas que los alumnos de inglés deben manejar al leer en este idioma, lo cual 
se evidencia en el siguiente segmento de entrevista: "Los contenidos podrían ser respecto a la cultura, a tradiciones, a países, personas en las que puedan buscar inspiración, artistas, escritores famosos que ellos no conozcan mucho. Estos temas pueden ser también basados en nuestro país" (E02 [20:20]). En esta línea, los temas también son mencionados por otro de los entrevistados: "El contenido tendría que ser referido al tema de la unidad. Por ejemplo, si la unidad es sobre la familia, el texto debería tener vocabulario sobre esto para que el alumno pueda comprender lo que se indica" (E04 [18:18]).

En el marco de contenidos asociados a las creencias de los estudiantes de pedagogía sobre la habilidad de comprensión lectora en inglés, ellos también se refirieron a los tiempos verbales, que son un tipo de contenido conceptual ligado a formas gramaticales usadas para expresar acciones en presente, pasado o futuro. En este sentido, uno de los informantes plantea que: "Deberían enseñarse las formas básicas de los tiempos verbales. Así los alumnos logran entender lo que están leyendo. Si es que leen una historia, pueden comprender lo que pasó y cuál fue el desarrollo" (E09 [19:19]). Otro de los participantes hace un comentario similar: "Un contenido importante es identificar los tiempos verbales porque mucha gente lee historias, pero después no sabe cuándo ocurrieron" (E10 [21:21]).
Respecto a las creencias de los futuros docentes sobre contenidos asociados a la habilidad de comprensión lectora en inglés, los participantes también aluden a contenidos procedimentales, basados en el desarrollo de estrategias de comprensión lectora. En este orden de ideas, cuando se le preguntó a una de las informantes sobre los contenidos que se deberían incluir en una clase, respondió: "Se deberían enseñar estrategias para que los alumnos aprendan a leer o entender un texto. Uno sólo lee sin aplicar técnicas" (E01 [19:19]). Asimismo, otro de los informantes alude a estos contenidos procedimentales, haciendo alusión a una estrategia de lectura vinculada con la comprensión global de un texto: "Los alumnos deben entender el sentido del texto completo más que solo la traducción literal de las palabras" (E06 [15:15]).

\section{Creencias de futuros profesores de inglés sobre actividades y recursos relacionados con la habilidad de comprensión lectora}

La categoría Creencias de futuros profesores de inglés sobre actividades relacionadas con la habilidad de comprensión lectora, que se ilustra en la figura 2, permite identificar la subcategoría actividades durante la lectura. Por otro lado, la categoría Creencias de futuros profesores de inglés sobre recursos relacionados con la habilidad de comprensión lectora, expuesta también en la figura 2, presenta las subcategorías textos no literarios y textos literarios.

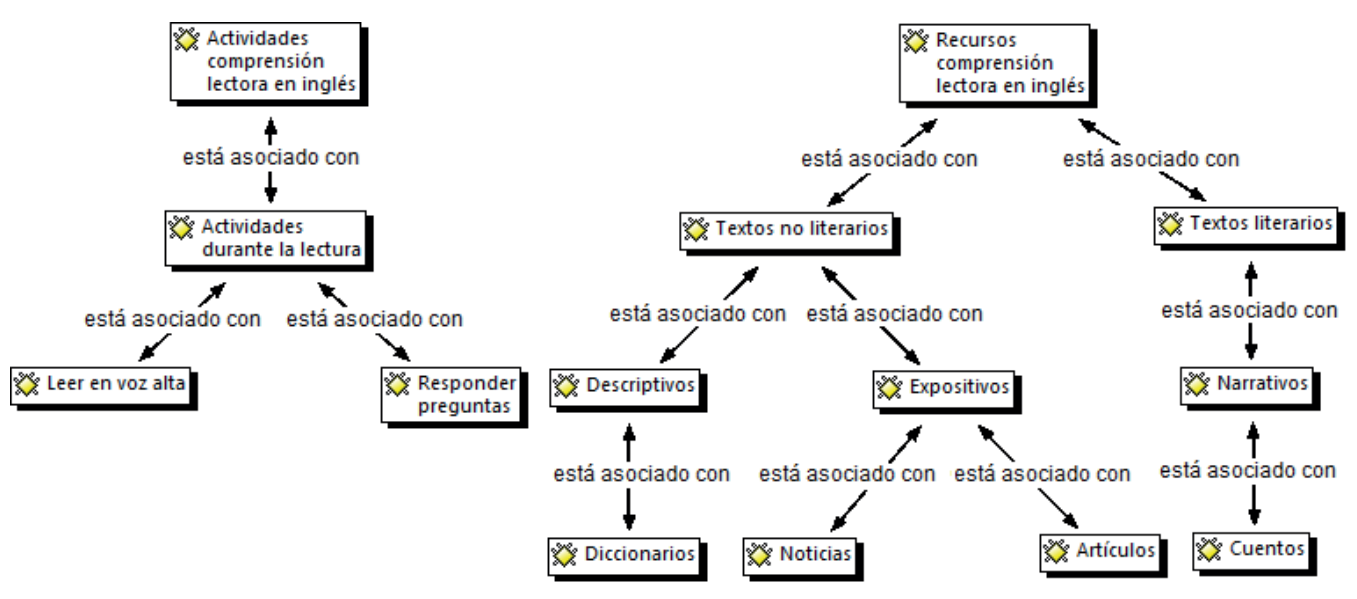

Figura 2. Red conceptual asociada a Creencias de futuros profesores de inglés sobre actividades y recursos relacionados con la habilidad de comprensión lectora.

Fuente: elaboración propia. 
En el marco de las creencias que poseen los futuros docentes sobre actividades de comprensión lectora en inglés, ellos aluden a aquellas realizadas durante la lectura en una clase de lengua extranjera. De acuerdo con lo planteado por los informantes, una de estas corresponde a leer en voz alta, perspectiva que se ilustra a continuación: "Nadie hace leer a los alumnos en voz alta. Ellos sí quieren aprender, pero es uno como profesor que no les da las oportunidades. Creo que esto se debería realizar para hacerlos participar más en la clase" (E01 [23:23]). Asociado al punto previo, otro de los entrevistados indica la misma tarea, refiriéndose a lo llevado a cabo en el sistema escolar: "Otra actividad podría ser leer en voz alta. En las escuelas siempre hacen lo mismo. Si los alumnos están leyendo, el profesor le solicita a otro estudiante que continúe con la lectura. Eso permite saber si él o ella está siguiendo el texto" (E03 [21:21]).

Otra de las actividades realizadas durante la lectura explicitadas por los estudiantes que participaron en el estudio fue responder preguntas sobre la información presentada en los textos leídos. Al respecto, uno de los entrevistados comenta: "Las típicas actividades son leer textos y responder preguntas sobre información general o específica. Esto para comprobar si los estudiantes comprendieron el texto o no" (E06 [23:23]). Asimismo, otro de los participantes parece plantear una perspectiva equivalente: "Una hoja de trabajo debe haber preguntas para que los alumnos las respondan. Estas los ayudarán al entender el texto que leyeron" (E05 [27:27]).

Por otro lado, considerando las creencias de los futuros profesores de inglés vinculadas con el uso de recursos que se deben emplear en una clase de comprensión lectora, ellos aluden a diferentes textos que facilitan el aprendizaje y la enseñanza de esta habilidad en la lengua extranjera. De acuerdo con sus respuestas, estos se asocian, en primer lugar, a los no literarios, que buscan proveer información a un lector sobre hechos de la vida cotidiana. En este marco, se destacan textos descriptivos que corresponden a los diccionarios. Estos recursos, de acuerdo con los entrevistados, ayudarían a los aprendices de la lengua inglesa a solucionar ciertos inconvenientes relacionados con su conocimiento de vocabulario en inglés ya que incluyen el significado de las palabras desconocidas. Al respecto, uno de los estudiantes de pedagogía manifiesta: "Creo que se aprende practicando, leyendo harto. También buscando, en el diccionario, las palabras que no se entiendan al leer, pero que estén dentro de un contexto" (E11 [05:05]). En función del mismo punto de vista, a pesar de que los entrevistados plantean el uso de diccionarios, uno de ellos manifiesta debilidades experimentadas por aprendices de la lengua inglesa al emplear los mismos: "Los alumnos muchas veces llegan a séptimo año y no saben ocupar un diccionario. Ellos invierten tiempo en aprenderlo a usar, luego en cómo buscar palabras. Si el diccionario está sólo en inglés, no entienden el significado en sî" (E09 [33:33]).

En función de los textos no literarios que mencionan los participantes, se destacan también los de carácter expositivo, que son útiles para presentar información sobre diferentes temáticas relacionadas con la realidad, por ejemplo las noticias, como se ejemplifica en el siguiente segmento de entrevista: "Se deberían emplear noticias de la actualidad. Puede resultar interesante leer algo actual que está pasando al otro lado del mundo o lo que está pasando en nuestro país, pero redactado por un diario de habla inglesa" (E13 [34:34]). Respecto a este punto de vista, otro de los informantes también asevera: "Yo usaría noticias para enseñar a otros a leer. Estas presentan información real, es parte de su vida cotidiana o lo que sus papás leen todos los días" (E02 [36:36]).

Otros textos expositivos que los futuros docentes de inglés vinculan con la habilidad de comprensión lectora en inglés son artículos sobre diferentes temas. Esta visión se explicita a través del siguiente segmento de entrevista: "Los alumnos deben leer artículos... estos abarcan cada tema que se está estudiando. Si se está revisando tecnología, ellos van a leer algo más científico" (E08 [31:31]). Al respecto, otro de los participantes plantea que el uso de este tipo de texto se asocia a una determinada edad de los aprendices: "Depende del nivel de los estudiantes y la edad. Para adolescentes o adultos sería más atractivo un artículo, algo que sea más comprensible para personas de esas edades" (E07 [33:33]). 
Asociado a las creencias de los participantes sobre recursos respecto a la habilidad de comprensión lectora en inglés, ellos indican, por otro lado, el uso de textos literarios. Según ellos, se deberían incluir, en una clase de lectura, textos narrativos, tales como cuentos, que relatan sucesos ficticios o reales que son realizados por personajes. Evidencia de esto se presenta en el siguiente segmento de entrevista, donde uno de los estudiantes de pedagogía alude a generar la motivación de los aprendices al enseñarles a leer en inglés: "Los textos van a depender de la edad de los estudiantes. Si ellos son más pequeños, se pudiesen usar cuentos de temáticas más fantásticas que les llamen la atención" (E05 [36:36]). En la misma línea, otro de los informantes, haciendo alusión al nivel de los alumnos, comenta que: "Primero, partiría con un texto de corta extensión como un cuento y después los haría leer textos más complejos" (E10 [38:38]).

\section{Creencias de futuros profesores de inglés sobre evaluación respecto a la habilidad de comprensión lectora}

La categoría Creencias de futuros profesores de inglés sobre evaluación respecto a la habilidad de comprensión lectora, la cual se ilustra en la figura 3, permite identificar las subcategorías evaluación formativa y evaluación sumativa.

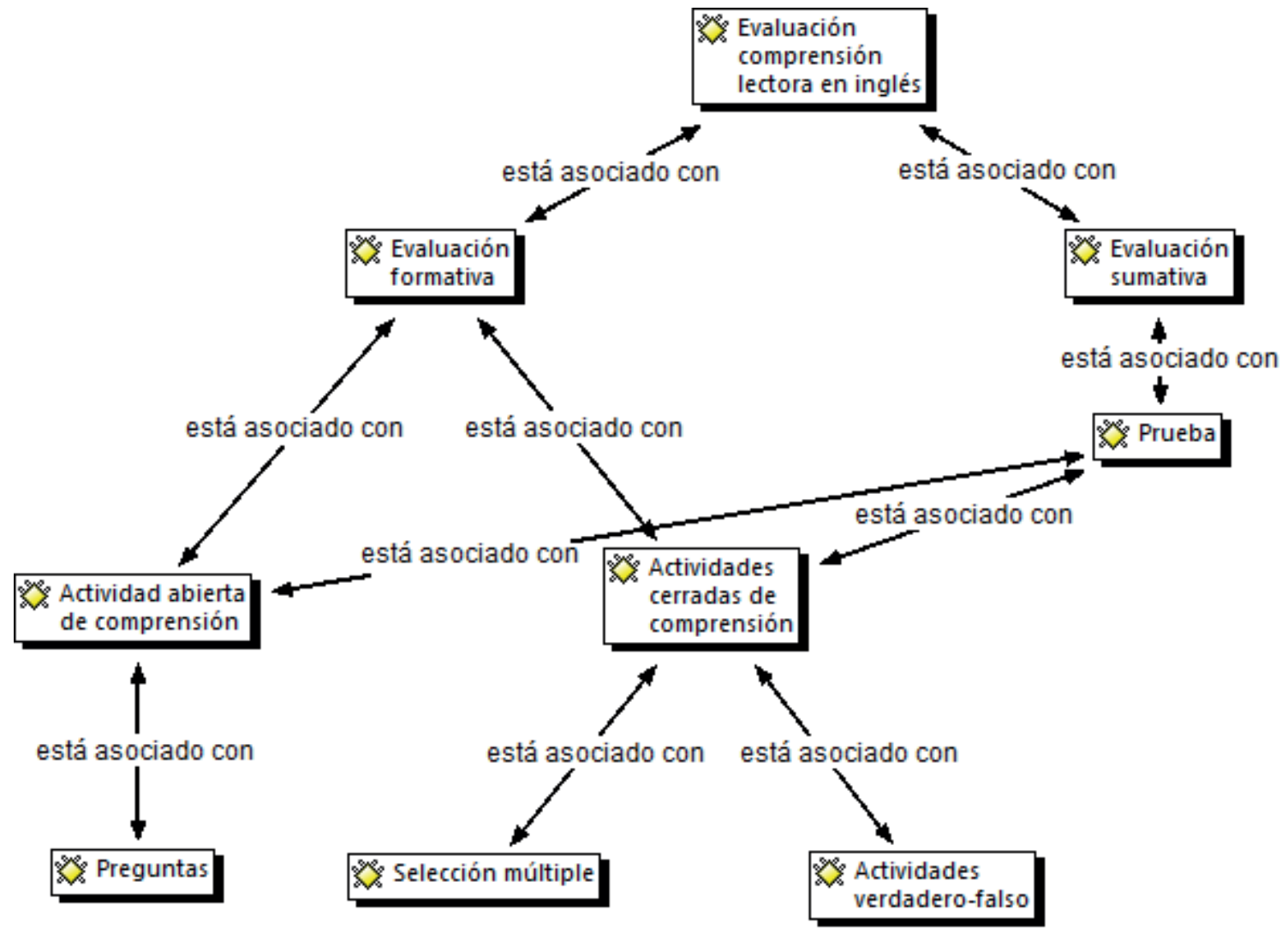

Figura 3. Red conceptual asociada a Creencias de futuros profesores de inglés sobre evaluación respecto a la habilidad de comprensión lectora.

Fuente: elaboración propia. 
En el marco de las creencias de los estudiantes de pedagogía en inglés sobre la evaluación asociada a la habilidad de comprensión lectora, ellos destacan, en primer lugar, la evaluación formativa. De acuerdo con lo que plantean, esta se lleva a cabo durante el proceso de enseñanza para identificar los aprendizajes construidos por los aprendices en el marco de esta habilidad. Esto puede hacerse por medio de una actividad abierta de comprensión, en la cual los alumnos pueden crear sus propias respuestas en función de lo comprendido en los textos leídos. Para los participantes, esta tarea se asocia específicamente a responder preguntas, lo cual se explicita en el siguiente segmento de entrevista: "En una clase, se puede evaluar empleando preguntas abiertas para que los alumnos reconozcan información del texto. Así nos damos cuenta sobre lo que ellos entendieron" (E09 [44:44]). Asimismo, otra de las estudiantes de pedagogía plantea por qué ella consideraría este tipo de actividad de evaluación: "Al enseñar comprensión lectora, yo evaluaría a través de preguntas. A mí me gustaría usar preguntas abiertas, aunque sean subjetivas, ya que los alumnos pueden demostrar lo que han realmente comprendido sin responder al azar" (E12 [34:34]).

En cuanto a la evaluación formativa respecto del aprendizaje de la habilidad de comprensión lectora en inglés, los participantes expresan, además, actividades cerradas de comprensión para identificar lo que los alumnos comprenden sobre los textos leídos en una clase. De acuerdo con los futuros docentes, estas presentan respuestas predeterminadas que los aprendices deben seleccionar. Una de estas actividades corresponde a los ítems de selección múltiple, lo cual se evidencia a continuación: "Los alumnos deben responder preguntas de selección múltiple en una clase. Estas debiesen ser respondidas después de las preguntas abiertas ya que ellos tienen que discriminar diferentes alternativas de acuerdo con el texto" (E04 [34:34]). En la misma línea, otras actividades cerradas de comprensión destacadas por los informantes para evaluar el aprendizaje de los alumnos respecto a esta habilidad son las que implican verdadero-falso. Lo anterior se menciona en el siguiente segmento de entrevista: "En las clases, se debe identificar el progreso de los alumnos al leer textos por medio de actividades que tengan verdadero o falso, en las que ellos vayan reconociendo y seleccionando lo que corresponde según lo que leyeron" (E08 [40:40]).

En segundo lugar, los futuros docentes de inglés manifiestan que se debe considerar la evaluación sumativa para calificar los aprendizajes construidos por los alumnos respecto a la habilidad de comprensión lectora en inglés. Consideran que esta debería implementarse al final de una unidad didáctica por medio de una prueba. Así lo afirma uno de los entrevistados: "La prueba es útil para evaluar al alumno al final del proceso. Esta puede incluir un texto parecido a los leídos en las clases" (E09 [49:49]). En esta línea, los informantes declaran que una prueba de comprensión lectora en inglés debería integrar tanto actividades abiertas de comprensión como cerradas. Esta visión se evidencia en el siguiente segmento de entrevista, donde uno de los estudiantes de pedagogía alude a cómo se evalúa el aprendizaje de esta habilidad en su formación universitaria: "Se debería evaluar como a nosotros. En la universidad, rendimos pruebas de comprensión lectora, donde debemos responder preguntas abiertas sobre textos para demostrar si los comprendemos o no. También respondemos ítems de multiple choice, verdadero-falso, unión de pares" (E05 [41:41]).

\section{Análisis de resultados}

En el marco de las creencias de los participantes sobre objetivos de la habilidad de comprensión lectora en inglés, ellos consideran que los estudiantes de inglés deberían identificar información de tipo general y específica en los textos leídos. Este tipo de ideas sobre el desarrollo de la habilidad puede asociarse al uso de dos estrategias de comprensión lectora, las cuales permiten al aprendiz construir significados relacionados con los textos leídos (véase, por ejemplo, Elabsy, 2013; Grabe y Stoller, 2013). Una de estas estrategias se denomina skimming y contribuye al propósito de reconocer información global, además del sentido y la organización del 
texto por medio de una lectura rápida. La otra estrategia es el scanning, que tiene como fin localizar información detallada o explícita.

Por otro lado, respecto a las creencias de los sujetos de estudio sobre los objetivos que se asocian a la habilidad de comprensión lectora en inglés, ellos plantean que se debe aprender contenidos lingüísticos, tales como vocabulario y gramática al leer en esta lengua extranjera. Este tipo de ideas vincularía la lectura con un proceso de decodificación del idioma, lo cual corresponde a una forma de procesamiento ascendente respecto a los textos leídos o de tipo bottom-up (Carrell, 1987). Esta lógica no ayudaría a los aprendices a desarrollar un proceso de comprensión de la lengua extranjera ya que se centra, principalmente, en el reconocimiento de componentes segmentados del idioma. Por lo tanto, este tipo de creencias podría obstaculizar el aprendizaje y la enseñanza de la comprensión lectora desde un punto de vista funcional de la lengua.

Considerando las creencias de los estudiantes de pedagogía enmarcadas en contenidos respecto a la habilidad de comprensión lectora en inglés, ellos se refieren a dos tipos de saberes que los alumnos deberían construir al desarrollar esta competencia. En primer lugar, están los saberes de tipo conceptual, entre los que se destacan temas presentes en los textos leídos. Esta visión estaría enmarcada en una forma de procesamiento descendente de la información o top-down (Dambacher, 2010; Smith, 1978). Al respecto, leer en inglés se percibiría como un proceso, en donde los aprendices activan sus conocimientos previos y hacen inferencias sobre un determinado tema presente en el texto. En este sentido, la visión señalada permitiría que los aprendices construyeran significados respecto al sentido de los textos.

Otro de los contenidos de tipo conceptual asociado a la habilidad de comprensión lectora en inglés que los participantes mencionaron son los tiempos verbales. Sus creencias sobre esta competencia se encuentran ligadas al manejo de estos contenidos estructurales de la lengua extranjera. Esta lógica se relacionaría, al igual que lo mencionado respecto a los objetivos conceptuales, con una forma de procesamiento de la información ascendente o bottom-up. Lo anterior privilegiaría el aprendizaje de gramática y vocabulario más que el desarrollo de esta habilidad receptiva en sí.

Respecto a lo planteado sobre contenidos de la habilidad de comprensión lectora en inglés, los informantes poseen creencias enfocadas en el conocimiento de estrategias asociadas a esta habilidad como un tipo de contenido procedimental. Este tipo de ideas se enmarcan en usar el idioma de manera funcional, donde los usuarios de la lengua poseen herramientas que les permiten leer y comprender textos de manera autónoma (Grabe, 2009). Considerando este punto, se ha planteado que incentivar a los aprendices de inglés a utilizar determinadas estrategias para comprender textos escritos en esta lengua podría ayudarlos a desarrollar esta habilidad (Zare y Othman, 2013). En esta línea, ellos podrían ser más autónomos en el proceso de comprensión, al ser capaces de interpretar la información de una forma más efectiva (Cekiso y Madikiza, 2014; Fan, 2010).

Las creencias que poseen los participantes sobre las actividades vinculadas con la habilidad de comprensión lectora en inglés se relacionan también con la lectura en voz alta. De acuerdo con lo señalado, esta tarea permitía promover la participación de los aprendices en las clases. Este tipo de actividad se enmarcaría en una forma de procesamiento ascendente de la información o bottom-up ya que se tiende a privilegiar el conocimiento de aspectos fonológicos de la lengua por sobre la construcción de significados en los textos leídos. Por esta razón, esta tarea no ayudaría a los alumnos a desarrollar la competencia de comprensión lectora en la lengua inglesa (Farrell, 2009; Nunan, 2015).

Por otro lado, en función de las creencias sobre actividades de comprensión lectora en inglés, los estudiantes de pedagogía declaran la relevancia de las preguntas abiertas, ya que en ellas los aprendices tienen la posibilidad de producir sus propias respuestas acerca de lo leído en un texto. Según los resultados de algunos estudios, estas tareas parecen 
beneficiar el desarrollo de esta habilidad ya que los aprendices pueden reconocer información en los textos leídos y procesarla, de manera activa, para generar una respuesta (Khoshsima y Pourjam, 2014; Ozuru, Briner, Kurby y McNamara, 2013).

Otra de las creencias de los estudiantes de Pedagogía en Inglés sobre la comprensión lectora tiene que ver con los recursos. De acuerdo con sus respuestas, estos pueden ser no literarios y literarios. Asociado a los del primer tipo, ellos consideran relevante el uso de diccionarios para que los alumnos busquen el significado de las palabras de vocabulario de los textos que desconocen. Esta visión se enmarca en una forma de procesamiento ascendente o bottom-up, la cual se basa en prácticas de decodificación (Carrell, 1987). Como se ha planteado, este tipo de prácticas pudiese impedir el proceso de construcción de significados de los textos leídos.

Por otro lado, en el marco de las creencias de los participantes sobre los recursos asociados a la habilidad de comprensión lectora en inglés, ellos aluden a otros textos no literarios, tales como noticias y artículos, que son de tipo expositivo. Asimismo, se destacan textos literarios, tales como cuentos (que son de tipo narrativo). Al respecto, se ha planteado que el hecho de solicitar a los estudiantes leer diferentes tipos de textos se enmarca en una propuesta de enseñanza y aprendizaje comunicativa, donde los aprendices tienen la posibilidad de procesar el idioma como lo harían en la vida diaria a través de un proceso de construcción de significados (Duff, 2014). Asimismo, la selección de textos basada en las necesidades e intereses de los aprendices puede ayudarlos a mejorar esta habilidad receptiva (Basallo, 2016).

En el marco de las creencias sobre la evaluación de la comprensión lectora que poseen los estudiantes de pedagogía en inglés, ellos plantean la evaluación formativa. De acuerdo con sus respuestas, esta debería llevarse a cabo por medio de una actividad abierta de comprensión, la cual corresponde a preguntas, y actividades cerradas de comprensión, tales como actividades de selección múltiple y de verdadero-falso. En este sentido, se ha establecido que este tipo de tareas serían apropiadas para evaluar la comprensión de lectura ya que las mismas buscarían evidenciar el desarrollo de la habilidad receptiva por medio de la aplicación de estrategias de lectura (Alderson, 2000; Brown y Abeywickrama, 2010).

Por otro lado, considerando también las creencias de los participantes sobre la evaluación de la comprensión lectora en inglés, los estudiantes de pedagogía se refieren a la evaluación sumativa, la cual involucra la prueba e integra tanto actividades abiertas como actividades cerradas de comprensión. En esta línea, los informantes se refieren a un solo instrumento, que suele catalogarse como una forma de carácter tradicional ya que no permite a los aprendices solucionar inconvenientes de la vida cotidiana por medio de las cuatro habilidades de la lengua (Richard-Amato, 2010). Según otros estudios, es posible que este tipo de creencias sobre la evaluación de la comprensión lectora se hayan construido a través de las experiencias como alumnos del sistema escolar vivenciadas por los participantes (véase, por ejemplo, Erkmen, 2014 y Mak, 2011).

En resumen, los resultados indican que los estudiantes de Pedagogía en Inglés que participaron en esta investigación parecen poseer creencias sobre el aprendizaje y la enseñanza de la habilidad de comprensión lectora basadas en las formas de procesamiento de tipo ascendente (bottom-up) y descendente (top-down). En este marco, es importante señalar que en el desarrollo de la habilidad ambas formas de procesamiento interactúan en el proceso de construcción de significado de los textos.

Como han mencionado los autores en otros estudios (Tagle et al., 2017a, 2017b), los estudiantes de pedagogía suelen poseer creencias sobre la enseñanza y/o el aprendizaje del inglés de tipo tradicional y comunicativo. En esta línea, se ha sugerido que los programas de formación docente motiven a los futuros profesores llevar a cabo prácticas metacognitivas sobre cómo se aprende y enseña el inglés, lo cual, en el contexto de la presente investigación debería enfocarse en el desarrollo de la habilidad de comprensión lectora en esa lengua. Lo anterior porque los estudiantes construyen conocimiento del contenido (inglés) y conocimiento pedagógico 
del contenido (didáctica) a través de la formación universitaria. Al respecto, la forma de enseñar de los docentes que imparten cursos focalizados en el desarrollo de la competencia comunicativa y los procesos metacognitivos asociados pueden favorecer no solo la construcción del conocimiento del contenido y del conocimiento pedagógico del contenido, sino la modificación de las creencias que orientan su actuar profesional. En otras palabras, aprender a aprender a leer en inglés para un estudiante que se está preparando como profesional de la educación en el área de especialidad involucraría no solo tomar conciencia sobre el procedimiento o las estrategias seleccionadas para construir el significado de un texto, sino también orientar su enseñanza considerando este aprendizaje. Lo anterior puede, a su vez, favorecer, el sistema educacional, formando lectores que emplean diferentes estrategias para comprender la lengua extranjera.

\section{Conclusión}

Los participantes de este estudio expresan en sus respuestas creencias sobre la enseñanza y el aprendizaje de la habilidad de comprensión lectora en inglés, las cuales se basan en una forma de procesamiento ascendente (bottom-up) y, por otro lado, creencias centradas una forma de procesamiento descendente (top-down). En este sentido, se sugiere que los programas de formación de docentes de inglés se focalicen en generar situaciones de enseñanza y aprendizaje que permitan a los futuros maestros configurar una visión sobre el desarrollo de esta habilidad en la cual se promueva el proceso de construcción de significado de los textos leídos activando distintos tipos de conocimiento (lingüísticos, temáticos, discursivos, entre otros) desde una perspectiva estratégica. Para esto, se requiere motivar a los futuros docentes a llevar a cabo prácticas metacognitivas asociadas a cómo se desarrolla esta habilidad receptiva para, así, favorecer, en forma progresiva, la transformación de las creencias tradicionales por creencias que tengan un mayor impacto al enseñar y aprender a leer en inglés.

\section{Referencias}

Agencia de Calidad de la Educación (2015). Síntesis resultados de aprendizaje III educación media: Simce 2014 inglés. http://archivos.agenciaeducacion.cl/ resultados-2014/Sintesis_Resultados_IIIM_2014.PDF.

Alderson, J. C. (2000). Assessing reading. Cambridge: Cambridge University Press.

Alonso, I. y Fernández, M. (2003). Literature at the core of EFL lessons: A practical proposal. En R. Varela (ed.), All about teaching English: A course for teachers of English (pre-school to secondary) (pp. 319-337). Madrid: Editorial Centro de Estudios Ramón Erices.

Bachman, L. y Palmer, A. (2010). Language assessment in practice. Oxford: Oxford University Press.

Basallo, J. S. (2016). Adult EFL reading selection: Influence on literacy. Profile, Issues in Teachers' Professional Development, 18(1), 167-181.

Blázquez, F. y Tagle, T. (2010). Formación docente: un estudio de las creencias de alumnos y profesores sobre el proceso de enseñanza y aprendizaje del inglés. Revista Iberoamericana de Educación, 54(4), $1-12$.

Borg, S. (2006). Teacher cognition and language education: Research and practice. Londres/Nueva York, NY: Continuum.

Borg, S. (2011). The impact of in-service teacher education on language teachers' beliefs. System, 39(3), 370-380.

Brown, H. D. y Abeywickrama, P. (2010). Language assessment: Principles and classroom practices (2. ${ }^{\mathrm{a}}$ ed.). Thousand Oaks, CA: Pearson.

Canale, M. (1983). From communicative competence to communicative language pedagogy. En J.C. Richards y R. Schmidt (eds.), Language and communication (pp. 2-27). Londres: Longman.

Canale, M. y Swain, M. (1980). Theoretical bases of communicative approaches to second language teaching and testing. Applied Linguistics, 1(1), 1-47.

Carrell, P. L. (1987). Content and formal schemata in ESL reading. TESOL Quarterly, 21, 461-481.

Cekiso, M. y Madikiza, N. (2014). Reading strategies used by grade 9 English second language learners in a selected school. Reading \& Writing, 5(1), 1-7. 
Celce-Murcia, M., Dörnyei, Z. y Thurrell, S. (1995). Communicative competence: A pedagogically motivated model with content specifications. Issues in Applied Linguistics, 6(2), 5-35.

Celce-Murcia, M. y Olshain, E. (2000). Discourse and context in language teaching: A guide for language teachers. Cambridge: Cambridge University Press.

Consejo de Europa (2001). Marco Común Europeo de Referencia para las lenguas: aprendizaje, enseñanza y evaluación. Madrid: Instituto Cervantes/Ministerio de Educación, Cultura y Deporte/Anaya.

Cross, D. I. (2009). Alignment, cohesion, and change: Examining mathematics teachers' belief structures and their influence on instructional practices. Journal of Mathematics Teacher Education, 12(5), 325-346.

Dambacher, M. (2010). Bottom-up and top-down processes in reading. Potsdam: Universitätsverlag Potsdam.

Díaz, C., Alarcón, P. y Ortiz, M. (2012). El profesor de inglés: sus creencias sobre la evaluación de la lengua inglesa en los niveles primario, secundario y terciario. Íkala, Revista de Lenguaje y Cultura, 17(1), 15-26.

Díaz, C., Alarcón, P. y Ortiz, M. (2015). A case study on EFL teachers' beliefs about the teaching and learning of English in public education. Porta Linguarum, 23, 171-186.

Díaz, C. y Solar, M. I. (2008). Una mirada al sistema de creencias del docente de inglés universitario: un estudio de caso en una universidad chilena. Revista Electrónica Diálogos Educativos, 16(8), 50-74.

Duff, P. A. (2014). Communicative language teaching. En M. Celce-Murcia, D. M. Brinton y M. A. Snow (eds.), Teaching English as a second or foreign language (4. ${ }^{a}$ ed.) (pp. 15-30). Boston, MA: National Geographic Learning.

Elabsy, T. (2013). Successful reading strategies for second language learners: Theory and practice. Parker, co: Outskirts Press.

Erkmen, B. (2014). Novice EFL teachers' beliefs about teaching and learning, and their classroom practices. H. U. Journal of Education, 29(1), 99-113.

Ertmer, P. A. (2005). Teacher pedagogical beliefs: The final frontier in our quest for technology integration? Educational Technology Research and Development, 53(4), 25-39.
Fan, Y. C. (2010). The effect of comprehension strategy instruction on EFL learners' reading comprehension. Asian Social Science, 6(8), 19-29.

Farrell, T. S. C. (2009). Teaching reading to English language learners: A reflective guide. Londres: Corwin Press.

Goodman, K. S. (1988). The reading process. En P. L. Carrell, J. Devine y D. E. Eskey (eds.), Interactive approaches to second language reading (pp. 11-21). Cambridge: Cambridge University Press.

Grabe, W. (2009). Reading in a second language: Moving from theory to practice. Nueva York, NY: Cambridge University Press.

Grabe, W. y Stoller, F. L. (2013). Teaching and researching reading. Abingdon/Nueva York, NY: Routledge.

Grabe, W. y Stoller, F. L. (2014). Teaching reading for academic purposes. En Celce-Murcia, M., D. M. Brinton y M. A. Snow (Eds.), Teaching English as a second or foreign language (4. ${ }^{\mathrm{a}} \mathrm{ed}$.) (pp. 189-205). Boston, MA: National Geographic Learning.

Hedgcock, J. S. y Ferris, D. R. (2009). Teaching readers of English: students, texts, and contexts. Nueva York, NY: Routledge.

Inceday, V. y Kesli, Y. (2011). Foreign language learners' beliefs about grammar instruction and error correction. Procedia-Social and Behavioral Sciences, 15, 3394-3398.

Katz, A. (2014). Assessment in second language classrooms. En M. Celce-Murcia, D. M. Brinton y M. A. Snow (eds.), Teaching English as a second or foreign language (pp. 320-229). Boston, MA: National Geographic Learning.

Khoshsima, J. y Pourjam, F. (2014). A comparative study on the effects of cloze tests and open-ended questions on reading comprehension of Iranian intermediate EFL learners. International Journal on Studies in English Language and Literature (IJSELL), 2(7), 17-27.

Kusiak, M. (2013). Reading comprehension in Polish and English: Evidence from an introspective study. Cracovia: Jagiellonian University Press.

Levin, B. y He, Y. (2008). Investigating the content and sources of teacher candidates' personal practical theories. Journal of Teacher Education, 59(1), 55-68.

López, J. (2005). Planificar la formación con calidad. Madrid: Cisspraxis. 
Mak, S. H. (2011). Tensions between conflicting beliefs of an EFL teacher in teaching practice. RELC Journal, 42(1), 53-67.

Makalela, L. (2015). Translanguaging principles in L2 reading instruction: Implications for ESL pre-service teachers programme. En T. S. C. Farrell (ed.), International perspectives on English language teaching education: Innovations from the field (pp. 107-124). Nueva York, NY: Palgrave Macmillan.

Maley, A. (2011). Squaring the circle-reconciling materials as constraint with materials as empowerment. En B. Tomlinson (ed.), Materials development in language teaching (2. ${ }^{\mathrm{a}}$ ed.) (pp. 379-402). Cambridge: Cambridge University Press.

Ministerio de Educación de Chile (2012). Bases curriculares: Idioma extranjero inglés. Santiago: Ministerio de Educación.

Ministerio de Educación de Chile (2014). Estándares de inglés. http://portales.mineduc.cl/index2.php?id_ portal=49\&id_seccion=3276\&id_contenido $=13307$.

Mullis, I. V. S., Kennedy, A. M., Martin, M. O. y Sainsbury, M. (2006). Pirls 2006: marcos teóricos y especificaciones de evaluación (2. ${ }^{\text {e }}$ d). Madrid: Inecse, Ministerio de Educación y Ciencia.

Nunan, D. (2015). Teaching English to speakers of other languages: An introduction. Nueva York, NY: Routledge.

Oakhill, J., Cain, K. y Elbro, C. (2015). Understanding and teaching reading comprehension: A handbook. Abingdon: Routledge.

Ozuru, Y., Briner, S., Kurby, C. A. y McNamara, D. S. (2013). Comparing comprehension measured by multiple-choice and open-ended questions. Canadian Journal of Experimental Psychology, 67(3), 215-227.

Pajares, F. (1992). Teachers' beliefs and educational research: Cleaning up a messy construct. Review of Educational Research, 62(3), 307-332.

Phillips, J. C. y Pulliam Phillips, P. (2008). Beyond learning objectives: Develop measurable objectives that link to the bottom line. Alexandria, vA: The American Society for Training and Development.

Ramírez, P., Rossel, K. y Nazar, G. (2015). Comprensión lectora y metacognición: análisis de las actividades de lectura en dos textos de estudio de la asignatura de Lenguaje y Comunicación de séptimo año básico. Estudios Pedagógicos, 41(2), 213-231.
Richards, J. C. (2015). Key issues in language teaching. Cambridge: Cambridge University Press.

Richards, J. C. y Farrell, T. (2005). Professional development for language teachers. Cambridge: Cambridge University Press.

Richard-Amato, P. A. (2010). Making it happen: From interactive to participatory language teaching ( $4 .^{\mathrm{a}} \mathrm{ed}$.). White Plains, NY: Pearson Education.

Richardson, V. (2003). Preservice teachers' beliefs. En J. Raths y A. McAninch (eds.), Teacher beliefs and classroom performance: The impact of teacher education (pp. 1-22). Greenwich, CT: Information Age Publishing.

Rodríguez, S. (2012). Lengua y literatura: pruebas de acceso ciclos formativos de grado superior. Madrid: Paraninfo.

Sandín, M. (2003). Investigación cualitativa en educación: fundamentos y tradiciones. Madrid: McGraw-Hill.

Savignon, S. J. (2001). Communicative language teaching for the twenty-first century. En M. Celce-Murcia (ed.), Teaching English as a second or foreign language (3. ${ }^{\mathrm{a}}$ ed.) (pp. 13-28). Boston: Heinle \& Heinle.

Smith, F. (1978). Understanding reading (2. ${ }^{\text {a }}$ ed). Nueva York, NY: Holt, Rinehart, and Winston.

Stake, R. (1998). Investigación con estudios de casos. Madrid: Morata.

Tagle, T., Díaz, C., Alarcón, P., Quintana, M. y Ramos, L. (2014a). Creencias sobre el aprendizaje del inglés en la formación inicial docente. Educere, 18(61), 473-482.

Tagle, T., Díaz, C., Alarcón, P., Quintana, M. y Ramos, L. (2014b). Creencias de estudiantes de pedagogía sobre la enseñanza del inglés. Folios, 2(39), 77-87.

Tagle, T., Díaz, C., Alarcón, P., Quintana, M., Ramos, L. y Etchegaray, P. (2017a). Aprendizaje y enseñanza: creencias de estudiantes en formación de pedagogía en inglés. Páginas de Educación, 10(1), 64-90.

Tagle, T., Díaz, C., Alarcón, P., Ramos, L., Quintana, M. y Etchegaray, P. (2017b). Creencias sobre los roles del profesor y del estudiante que poseen futuros docentes de inglés en dos universidades chilenas. Folios, 45, 113-126.

Tomlinson, B. (2011). Glossary of basic terms for materials development in language teaching. En B. Tomlinson (ed.), Materials development in language teaching (2. ${ }^{\text {e ed. }) ~(p p . ~ 9-18) . ~ C a m b r i d g e: ~ C a m b r i d g e ~}$ University Press. 
Usó-Juan, E. y Martínez-Flor, A. (2006). Approaches to language learning and teaching: Towards acquiring communicative competence through the four skills. En E. Usó-Juan y A. Martínez-Flor (Eds.), Current trends in the development and teaching of the four language skills (pp. 3-26). Berlin: Mouton de Gruyter.

Xiao, L. (2015). What can we learn from classroom observations?: A study of CLT in a Chinese university context. En M. A. Vyas e Y. L. Patel (eds.), Teaching English as a second language: A new pedagogy for a new century (2. ${ }^{\text {a }}$ ed.) (pp. 7-44). Delhi: PHI Learning Private Limited.

Zabala, A. (2007). Los enfoques didácticos. En C. Coll, E. Martín, T. Mauri, M. Miras, J. Onrubia, I. Solé y A. Zabala (eds.), El constructivismo en el aula (17. ${ }^{\text {a }}$ ed.) (pp. 125-161). Barcelona: GRAó.

Zabala, A. y Arnau, L. (2014). Métodos para la enseñanza de las competencias. Barcelona: GRAó.

Zare, P. y Othman, M. (2013). The relationship between reading comprehension and reading strategy use among Malaysian ESL learners. International Journal of Humanities and Social Science, 3(13), 187-193.

\section{Anexo: Guion de entrevista}

1. ¿Cómo se aprende a comprender textos en inglés?

2. ¿Cómo se enseña a comprender textos en inglés?

3. ¿Qué objetivos deberían incluirse en una clase centrada en comprensión lectora en inglés? ¿Por qué deberían incluirse estos objetivos?

4. ¿Qué contenidos deberían incluirse en una clase centrada en comprensión lectora en inglés? ¿Por qué deberían incluirse estos contenidos?

5. ¿Qué actividades deberían incluirse en una clase centrada en comprensión lectora en inglés? ¿Por qué deberían incluirse estas actividades?

6. ¿Qué recursos/materiales deberían incluirse en una clase centrada en comprensión lectora en inglés? ¿Por qué deberían incluirse estos recursos/materiales?

7. ¿Qué tipos de textos deberían incluirse en una clase centrada en comprensión lectora en inglés? ¿Por qué deberían incluirse este tipo de textos?

8. ¿Qué se evalúa en la comprensión lectora de inglés?

9. ¿Cómo se evalúa la comprensión de lectura en inglés? ¿Qué instrumentos/procedimientos se deberían utilizar para evaluar la comprensión de lectura en inglés? ¿Por qué debería utilizarse estos instrumentos/ procedimientos?

10. ¿Cuándo (en qué momentos) se evalúa la comprensión lectora? 